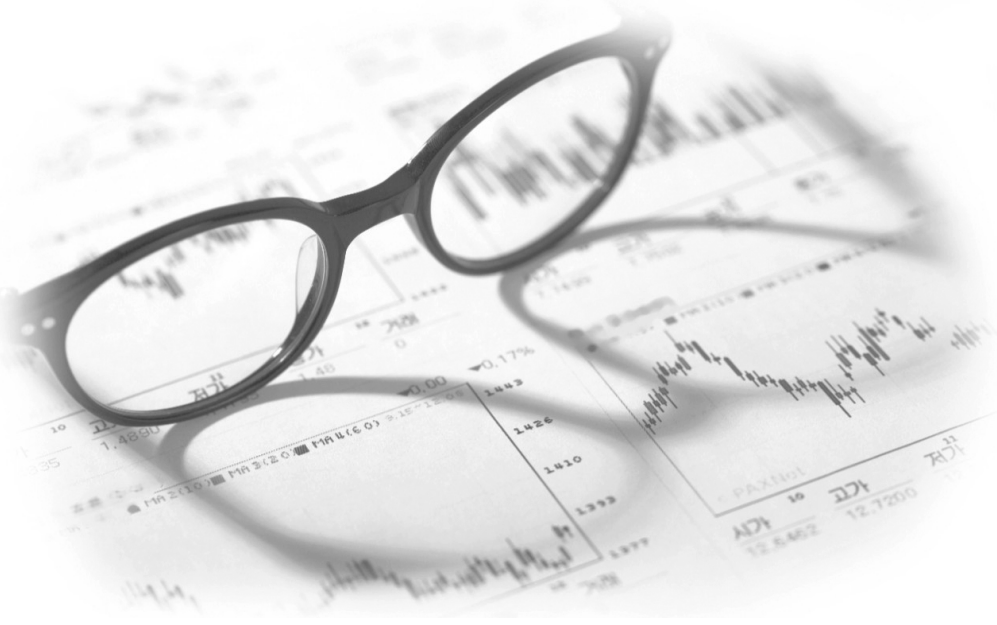

BLOOD RESEARCH

Volume $56 \cdot$ Number $3 \cdot$ September 2021

https://doi.org/10.5045/br.2021.2021126

Perspective

\section{Platelet count as an important prognostic factor for vaccine-induced immune thrombotic thrombocytopenia}

\author{
Soo-Mee Bang, M.D., Ph.D. ${ }^{1}$, \\ Sang-Hoon Na, M.D., Ph.D.', \\ Ji Hyun Kim, M.D., Ph.D. ${ }^{3}$, Seo Ree Kim, M.D. , \\ Seong-Rim Kim, M.D., Ph.D. ${ }^{5}$, \\ Seongsoo Jang, M.D., Ph.D. ${ }^{6}$
}

Department of Internal Medicine, ${ }^{1}$ Seoul National University
Bundang Hospital, Seoul National University College of
Medicine, Seongnam, ${ }^{2}$ Seoul National University Hospital,
Seoul, ${ }^{3}$ Department of Neurology, Korea University Guro
Hospital, Seoul, ${ }^{4}$ Department of Internal Medicine, College of
Medicine, The Catholic University of Korea, Seoul,
${ }^{5}$ Department of Neurosurgery, Bucheon St. Mary's Hospital,
College of Medicine, The Catholic University of Korea,
Bucheon, ${ }^{6}$ Department of Laboratory Medicine, Asan Medical
Center, University of Ulsan College of Medicine, Seoul, Korea

Received on July 6, 2021; Accepted on July 21, 2021

\section{Introduction}

Vaccine-induced immune thrombotic thrombocytopenia (VITT) has been reported in a patient after receiving two vaccines with a recombinant adenoviral vector encoding the spike glycoprotein of coronavirus disease (COVID-19) in Europe and the United States [1, 2]. Among approximately 12.7 million people who received ChAdOx1 nCov-19 (AstraZeneca) vaccines in Korea [3], we detected two cases of cerebral venous sinus thrombosis together with thrombocytopenia and positive results for platelet factor 4 (PF4)heparin antibody using enzyme-linked immunosorbent assay (ELISA). The fatality rate in our study was $50 \%$. This study aimed to analyze a suitable prognostic factor through a central review of domestic cases and a literature review of overseas reports.

\section{Clinical and laboratory characteristics of 44 VITT patients}

We reviewed two Korean patients and previously reported 42 patients [1, 4-6] with VITT onset after receiving AstraZeneca vaccines in this study. We excluded one reported case from England because PF4-heparin antibody was not detected using two ELISA methods [5]. In total, 44 patients were selected for inclusion in this analysis (Table 1 ), composed of 17 and 27 male and female patients, respectively, showing a female predominance, and with a median age of 36 years (range, 21-77 yr). Initial symptoms appeared at a median of 10 days after vaccination (range, 5-24 days). Symptoms were analyzed in only 10 patients; headache was the most common symptom related to cerebral venous thrombosis (CVT). CVT was detected in 29 patients, including the 2 Korean cases, which was the most frequent site in $66 \%$ of the total patients. Six patients showed combined cerebral hemorrhage among 29 CVT cases. Pulmonary embolism was diagnosed in 12 (27\%) patients, and arterial and splanchnic vein thromboses were detected in $10(23 \%)$ and $8(18 \%)$ patients, respectively.

All cases showed decreased platelet counts and elevated D-dimer levels, both at initial presentation $[5,6]$ and during treatment $[1,4,6]$. The initial and nadir platelet counts were available for 26 and 21 cases, respectively. The median platelet counts (range) initially and at nadir were 35,000 $(7,000-113,000)$ and 23,000 (8,000-107,000) (reference range, $150,000-440,000 / \mu \mathrm{L})$. All except two cases had positive results for PF4-heparin antibody on ELISA, but 
Table 1. Clinical and laboratory characteristics of patients with vaccine-induced immune thrombotic thrombocytopenia (VITT).

\begin{tabular}{|c|c|c|c|c|c|c|c|c|c|c|c|c|c|c|c|c|c|c|}
\hline $\begin{array}{l}\text { Patient } \\
\text { No. }\end{array}$ & $\begin{array}{l}\text { Age, } \\
\text { years }\end{array}$ & Sex & $\begin{array}{l}\text { Symptom } \\
\text { onset } \\
\text { ( } \mathrm{N} \text { of } \\
\text { days after } \\
\text { vaccination) }\end{array}$ & Symptoms & CVT & $\begin{array}{l}\text { Splan- } \\
\text { chnic } \\
\text { vein } \\
\text { throm- } \\
\text { bosis }^{\text {a) }}\end{array}$ & $\begin{array}{l}\text { Pulm- } \\
\text { onary } \\
\text { embo- } \\
\text { lism }\end{array}$ & $\begin{array}{l}\text { Other throm- } \\
\text { bosis or } \\
\text { hemorrhage }\end{array}$ & $\begin{array}{l}\text { Platelet } \\
\text { initial } \\
(\text { per } \mu \mathrm{L})\end{array}$ & $\begin{array}{l}\text { Platelet } \\
\text { nadir } \\
\text { (per } \mu \mathrm{L} \text { ) }\end{array}$ & $\begin{array}{l}\text { D-dimer, } \\
\text { peak } \\
(\mathrm{mg} / \mathrm{L})\end{array}$ & $\begin{array}{l}\text { INR } \\
\text { peak }\end{array}$ & $\begin{array}{l}\text { PTT } \\
\text { peak } \\
(\text { sec) }\end{array}$ & $\begin{array}{l}\text { Fibrino- } \\
\text { gen } \\
\text { nadir } \\
(\mathrm{mg} / \mathrm{dL})\end{array}$ & $\begin{array}{l}\text { PF4- } \\
\text { heparin } \\
\text { ELISA } \\
\text { (optical } \\
\text { density) }\end{array}$ & $\begin{array}{c}\text { Heparin } \\
\text { treatment }\end{array}$ & $\begin{array}{l}\text { Other } \\
\text { medical } \\
\text { conditions }\end{array}$ & Outcome \\
\hline Korean 1 & 33 & M & 11 & $\begin{array}{l}\text { Headache, } \\
\text { seizure }\end{array}$ & Yes & No & No & $\begin{array}{l}\text { Cerebral } \\
\text { hemorrhage }\end{array}$ & 77,000 & 65,000 & $>20$ & 1.01 & 35.7 & 197.9 & 3.10 & No & ACL-Ab & $\begin{array}{l}\text { Full } \\
\text { recovery }\end{array}$ \\
\hline Korean 2 & 33 & M & 8 & $\begin{array}{l}\text { Headache, } \\
\text { hemiparesis, } \\
\text { drowsiness }\end{array}$ & Yes & No & No & $\begin{array}{l}\text { Cerebral } \\
\text { hemorrhage }\end{array}$ & 14,000 & 10,000 & $>35.2$ & 1.51 & 60.7 & 77 & 0.72 & No & No & Fatal \\
\hline Ref $1-1$ & NA & NA & 5 & $\begin{array}{l}\text { Chills, fever, } \\
\text { nausea, and } \\
\text { epigastric } \\
\text { discomfort }\end{array}$ & Yes & Yes & Yes & $\begin{array}{l}\text { Aortoiliac artery } \\
\text { thrombosis }\end{array}$ & NA & 13,000 & 142 & 1.4 & 41.6 & 78 & 3.16 & Yes & No & Fatal \\
\hline Ref $1-2$ & NA & NA & 6 & NA & No & No & Yes & No & NA & 107,000 & 1.8 & 1.12 & 29 & 568 & 3.08 & $\left.L_{M W H}\right)^{e}$ & No & Recovering \\
\hline Ref 1-3 & NA & NA & 9 & NA & Yes & No & No & No & NA & 60,000 & 13 & $\mathrm{NA}$ & NA & NA & 3.5 & Unknown & No & Unknown \\
\hline Ref $1-4$ & NA & NA & 7 & NA & Yes & No & No & No & NA & 9,000 & NA & 1.66 & 46.6 & NA & 3.4 & Yes & CND & Fatal \\
\hline Ref 1-5 & NA & NA & 13 & NA & Yes & Yes & Yes & $\begin{array}{l}\text { Right } \\
\text { intra-ventricu } \\
\text { lar, iliofemoral } \\
\text { vein, IVC } \\
\text { thrombi }\end{array}$ & NA & 23,000 & NA & 1.25 & 64.8 & 173 & 1.2 & Yes & $\begin{array}{l}\text { WWD-l; } \\
\text { FVL; } \\
\text { ACL-Abs }\end{array}$ & Recovering \\
\hline $\operatorname{Ref} 1-6$ & NA & NA & 7 & NA & Yes & No & No & No & NA & 75,000 & 2.6 & 1.05 & 23 & NA & NA & Unknown & No & Recovering \\
\hline Ref $1-7$ & NA & NA & 8 & NA & Yes & No & No & No & NA & 29,000 & $>33.0$ & 1.34 & 45 & 210 & NA & Yes & No & Recovering \\
\hline $\operatorname{Ref} 1-8$ & NA & NA & 8 & NA & Yes & No & No & $\begin{array}{l}\text { Widespread } \\
\text { microvascular } \\
\text { thrombi } \\
\text { (brain, lungs, } \\
\text { and kidneys) }{ }^{\text {d) }}\end{array}$ & NA & 16,000 & NA & $\mathrm{NA}$ & $\mathrm{NA}$ & NA & 2.02 & No & No & Fatal \\
\hline Ref $1-9$ & NA & NA & 16 & NA & Yes & No & No & $\begin{array}{l}\text { Multiple } \\
\text { organ } \\
\text { thrombi }^{\text {d) }}\end{array}$ & NA & 13,000 & 21 & 1.7 & 46.1 & 40 & 3.51 & No & No & Fatal \\
\hline Ref $1-10$ & NA & NA & 11 & NA & Yes & Yes & No & No & NA & 8,000 & $>35.0$ & NA & NA & 80 & 2.35 & No & No & Fatal \\
\hline Ref 1-11 & NA & NA & $12^{\mathrm{b})}$ & NA & Pending ${ }^{c}$ & No & No & $\begin{array}{l}\text { Cerebral } \\
\text { hemorrhage }^{c}\end{array}$ & NA & $\mathrm{NA}$ & NA & NA & $\mathrm{NA}$ & NA & 2.16 & No & Unknown & Fatal \\
\hline Ref $4-1$ & 37 & $\mathrm{~F}$ & 8 & $\begin{array}{l}\text { Fever, } \\
\text { headache, } \\
\text { visual } \\
\text { disturbances }\end{array}$ & Yes & No & No & No & NA & 22,000 & $>35$ & 1.2 & 25 & 210 & 3.7 & $\begin{array}{r}\text { Initial low } \\
\text { dose of } \\
\text { LMWH }\end{array}$ & NA & Fatal \\
\hline Ref $4-2$ & 42 & $\mathrm{~F}$ & 10 & $\begin{array}{l}\text { Headache, } \\
\text { drowsiness }\end{array}$ & Yes & No & No & No & NA & 14,000 & $>35$ & 1 & 31 & 80 & 35.93 .4 & $\begin{array}{l}\text { Reduced } \\
\text { dose of } \\
\text { LMWH }\end{array}$ & NA & Fatal \\
\hline Ref 4-3 & 32 & M & 7 & Back pain & No & Yes & No & $\begin{array}{l}\text { Azygos vein, } \\
\text { hemiazygos } \\
\text { vein, and } \\
\text { several } \\
\text { basivertebral } \\
\text { veins' } \\
\text { thrombi }\end{array}$ & NA & 10,000 & $>35$ & 1.1 & 25 & 230 & 3.6 & $\begin{array}{r}\text { Reduced } \\
\text { dose of } \\
\text { LMWH }\end{array}$ & NA & $\begin{array}{l}\text { Full } \\
\text { recovery }\end{array}$ \\
\hline Ref $4-4$ & 39 & $\mathrm{~F}$ & 10 & $\begin{array}{l}\text { Headache, } \\
\text { abdominal } \\
\text { pain }\end{array}$ & Yes & No & No & No & NA & 70,000 & 13 & 1.3 & 25 & 120 & 3.8 & $\begin{array}{l}\text { Reduced } \\
\text { dose of } \\
\text { LMWH }\end{array}$ & $\mathrm{NA}$ & $\begin{array}{l}\text { Full } \\
\text { recovery }\end{array}$ \\
\hline Ref 4-5 & 54 & $\mathrm{~F}$ & 7 & $\begin{array}{l}\text { Headache, } \\
\text { hemiparesis }\end{array}$ & Yes & No & No & No & $\mathrm{NA}$ & 19,000 & $>35$ & 1.1 & 29 & 120 & 2.9 & $\begin{array}{l}\text { Heparin } \\
(5,000 \\
\text { IU) }\end{array}$ & $\mathrm{NA}$ & Fatal \\
\hline Ref 5-1 & 30 & $\mathrm{~F}$ & 13 & NA & Yes & Yes & Yes & $\begin{array}{l}\text { Ischemic } \\
\text { bowel with } \\
\text { infarction }\end{array}$ & 27,000 & NA & NA & NA & NA & NA & Pos & NA & $\mathrm{NA}$ & Survived \\
\hline Ref 5-2 & 55 & $\mathrm{~F}$ & 6 & NA & No & Yes & No & $\begin{array}{l}\text { Acute aortic } \\
\text { thrombosis } \\
\text { and cerebral } \\
\text { hemorrhage }\end{array}$ & 11,000 & NA & NA & NA & NA & NA & Pos & NA & NA & Died \\
\hline Ref 5-3 & 26 & $\mathrm{~F}$ & 12 & NA & Yes & No & No & No & 64,000 & NA & NA & NA & NA & NA & 2.45 & NA & NA & Survived \\
\hline Ref 5-4 & 52 & $\mathrm{~F}$ & 10 & NA & Yes $^{\mathrm{d})}$ & No & $Y_{e s}{ }^{d)}$ & $\begin{array}{l}\text { Cerebral } \\
\text { hemorrhage }\end{array}$ & 31,000 & NA & NA & NA & NA & NA & 2.26 & NA & NA & Died \\
\hline Ref 5-5 & 38 & $M$ & 14 & NA & No & No & Yes & No & 16,000 & NA & NA & NA & $\mathrm{NA}$ & NA & 2.84 & NA & NA & Died \\
\hline Ref 5-6 & 49 & $\mathrm{~F}$ & 15 & NA & Yes & No & Yes & $\begin{array}{l}\text { Cerebral } \\
\text { hemorrhage }\end{array}$ & 14,000 & $\mathrm{NA}$ & NA & NA & NA & NA & Pos & NA & NA & Survived \\
\hline Ref 5-7 & 25 & M & 9 & NA & Yes & No & No & No & 19,000 & NA & NA & NA & NA & NA & Pos & NA & NA & Died \\
\hline Ref 5-8 & 32 & M & 19 & NA & Yes & No & No & No & 87,000 & NA & NA & NA & NA & NA & Pos & NA & NA & Survived \\
\hline Ref 5-9 & 35 & $\mathrm{~F}$ & 9 & NA & Yes & No & No & No & 65,000 & NA & NA & NA & NA & NA & Pos & $\mathrm{NA}$ & NA & Survived \\
\hline Ref 5-10 & 77 & M & 8 & NA & No & No & Yes & No & NA & NA & NA & NA & $\mathrm{NA}$ & NA & Pos & NA & NA & Survived \\
\hline Ref 5-11 & 66 & M & 12 & NA & No & No & No & $\begin{array}{l}\text { Deep vein } \\
\text { thrombosis, } \\
\text { adrenal } \\
\text { hemorrhage }\end{array}$ & 34,000 & NA & NA & NA & NA & NA & Pos & NA & NA & Survived \\
\hline Ref 5-12 & 34 & M & 14 & NA & Yes & No & No & No & 23,000 & NA & NA & NA & NA & NA & Pos & NA & NA & Survived \\
\hline Ref 5-13 & 54 & M & 10 & NA & No & Yes & No & $\begin{array}{l}\text { Myocardial } \\
\text { infarction }\end{array}$ & 71,000 & NA & NA & NA & NA & NA & 0.76 & NA & NA & Died \\
\hline
\end{tabular}


Table 1. Continued.

\begin{tabular}{|c|c|c|c|c|c|c|c|c|c|c|c|c|c|c|c|c|c|c|}
\hline $\begin{array}{l}\text { Patient } \\
\text { No. }\end{array}$ & $\begin{array}{l}\text { Age, } \\
\text { years }\end{array}$ & Sex & $\begin{array}{c}\text { Symptom } \\
\text { onset } \\
\text { ( } \mathrm{N} \text { of } \\
\text { days after } \\
\text { vaccination) }\end{array}$ & Symptoms & CVT & $\begin{array}{l}\text { Splan- } \\
\text { chnic } \\
\text { vein } \\
\text { throm- } \\
\text { bosis }^{\text {a) }}\end{array}$ & $\begin{array}{c}\text { Pulm- } \\
\text { onary } \\
\text { embo- } \\
\text { lism }\end{array}$ & $\begin{array}{l}\text { Other throm- } \\
\text { bosis or } \\
\text { hemorrhage }\end{array}$ & $\begin{array}{c}\text { Platelet } \\
\text { initial } \\
\text { (per } \mu \mathrm{L})\end{array}$ & $\begin{array}{l}\text { Platelet } \\
\text { nadir } \\
\text { (per } \mu \mathrm{L})\end{array}$ & $\begin{array}{c}\text { D-dimer, } \\
\text { peak } \\
(\mathrm{mg} / \mathrm{L})\end{array}$ & $\begin{array}{l}\text { INR } \\
\text { peak }\end{array}$ & $\begin{array}{l}\text { PTT } \\
\text { peak } \\
\text { (sec) }\end{array}$ & $\begin{array}{l}\text { Fibrino- } \\
\text { gen } \\
\text { nadir } \\
\text { (mg/dL) }\end{array}$ & $\begin{array}{c}\text { PF4- } \\
\text { heparin } \\
\text { ELISA } \\
\text { (optical } \\
\text { density) }\end{array}$ & $\begin{array}{c}\text { Heparin } \\
\text { treatment }\end{array}$ & $\begin{array}{c}\text { Other } \\
\text { medical } \\
\text { conditions }\end{array}$ & Outcome \\
\hline Ref 5-14 & 71 & $\mathrm{~F}$ & 14 & NA & No & No & No & $\begin{array}{l}\text { Hemorrhagic } \\
\text { symptoms } \\
\text { only }\end{array}$ & 17,000 & NA & NA & NA & $\mathrm{NA}$ & NA & Pos & NA & NA & Survived \\
\hline Ref 5-15 & 22 & $\mathrm{~F}$ & 10 & NA & Yes & No & No & $\begin{array}{l}\text { Cerebral } \\
\text { hemorrhage }\end{array}$ & 100,000 & NA & $\mathrm{NA}$ & NA & $\mathrm{NA}$ & NA & 1.4 & NA & NA & Died \\
\hline Ref 5-16 & 39 & $\mathrm{~F}$ & 10 & $\mathrm{NA}$ & No & No & No & $\begin{array}{l}\text { Cerebral } \\
\text { infarction }\end{array}$ & 57,000 & NA & $\mathrm{NA}$ & NA & NA & NA & 1.4 & $\mathrm{NA}$ & NA & Survived \\
\hline Ref 5-17 & 70 & $\mathrm{~F}$ & 17 & $\mathrm{NA}$ & No & No & Yes & $\begin{array}{l}\text { Deep vein } \\
\text { thrombosis }\end{array}$ & 28,000 & NA & NA & NA & NA & $\mathrm{NA}$ & Pos & $\mathrm{NA}$ & NA & Survived \\
\hline Ref 5-18 & 21 & M & 10 & NA & No & No & No & $\begin{array}{l}\text { Cerebral } \\
\text { infarction }\end{array}$ & 113,000 & NA & NA & NA & NA & NA & 2.8 & NA & NA & Survived \\
\hline Ref 5-19 & 46 & $\mathrm{~F}$ & 14 & NA & Yes & No & No & No & 7,000 & $\mathrm{NA}$ & NA & NA & NA & NA & $>3.00$ & NA & $\mathrm{NA}$ & Survived \\
\hline Ref 5-20 & 32 & $\mathrm{~F}$ & 12 & NA & Yes & No & No & No & 98,000 & NA & $\mathrm{NA}$ & NA & $\mathrm{NA}$ & NA & 2.17 & NA & $\mathrm{NA}$ & Died \\
\hline Ref 5-21 & 48 & M & 14 & NA & Yes & No & No & No & 16,000 & $\mathrm{NA}$ & NA & NA & NA & NA & 2.45 & NA & NA & Survived \\
\hline Ref 5-22 & 49 & $\mathrm{~F}$ & 24 & NA & No & No & Yes & No & 61,000 & $\mathrm{NA}$ & $\mathrm{NA}$ & NA & $\mathrm{NA}$ & NA & $>3.00$ & NA & $\mathrm{NA}$ & Survived \\
\hline Ref 6-1 & 72 & $\mathrm{~F}$ & 7 & $\begin{array}{l}\text { Leg } \\
\text { pain, } \\
\text { claudication }\end{array}$ & No & No & No & $\begin{array}{l}\text { Peripheral } \\
\text { artery } \\
\text { thromboses }\end{array}$ & 36,000 & 39,000 & $>20$ & NA & NA & 237 & 2.70 & Yes & NA & $\begin{array}{l}\text { Full } \\
\text { recovery }\end{array}$ \\
\hline Ref 6-2 & 63 & M & 18 & $\begin{array}{l}\text { Leg } \\
\text { clamping }\end{array}$ & No & No & Yes & $\begin{array}{l}\text { Peripheral } \\
\text { artery } \\
\text { thromboses, } \\
\text { deep vein } \\
\text { thrombosis }\end{array}$ & 36,000 & 26,000 & $>10$ & 1.3 & NA & 140 & 1.78 & Yes & NA & $\begin{array}{l}\text { Full } \\
\text { recovery }\end{array}$ \\
\hline Ref 6-3 & 69 & M & 12 & $\begin{array}{l}\text { Headache, } \\
\text { confusion }\end{array}$ & Yes & Yes & Yes & $\begin{array}{l}\text { Cerebral } \\
\text { infarction, } \\
\text { internal } \\
\text { jugular vein } \\
\text { thrombosis }\end{array}$ & 35,000 & 29,000 & 3.35 & 1.4 & NA & 210 & 2.69 & No & $\mathrm{NA}$ & Recovering \\
\hline
\end{tabular}

a) Splanchnic-vein thrombosis indicates thrombosis of the portal, mesenteric, splenic, or hepatic veins

${ }^{\text {b) }}$ The day when the body of the deceased was found.

${ }^{c}$ Brain neuropathological results were pending at time of this report; CVT had not been ruled out.

${ }^{d)}$ These were the postmortem findings.

${ }^{\text {e) } T r e a t m e n t ~ w i t h ~ l o w-m o l e c u l a r-w e i g h t ~ h e p a r i n ~ w a s ~ a s s o c i a t e d ~ w i t h ~ c l i n i c a l ~ i m p r o v e m e n t ~ a n d ~ i n c r e a s i n g ~ p l a t e l e t ~ c o u n t s ~(107,000 ~ t o ~ 132,000 ~ o v e r ~} 3$ days). The patient's drug was then switched to a direct oral anticoagulant when the ELISA showed positive results for antibodies against PF4-heparin, with further clinical and platelet-count recovery.

Abbreviations: ACL-Abs, anticardiolipinantibodies; CVT, cerebral venous (sinus) thrombosis of the cortical vein; ELISA, enzyme-linked immunosorbent assay; F, female; FVL, factor V Leiden; INR, international normalized ratio; IVC, inferior vena cava; LMWH, low-molecular-weight heparin; M, male; NA, not available; PF4, platelet factor 4; Pos, positive; PTT, partial thromboplastin time; VWD-I, type 1 von Willebrand disease.

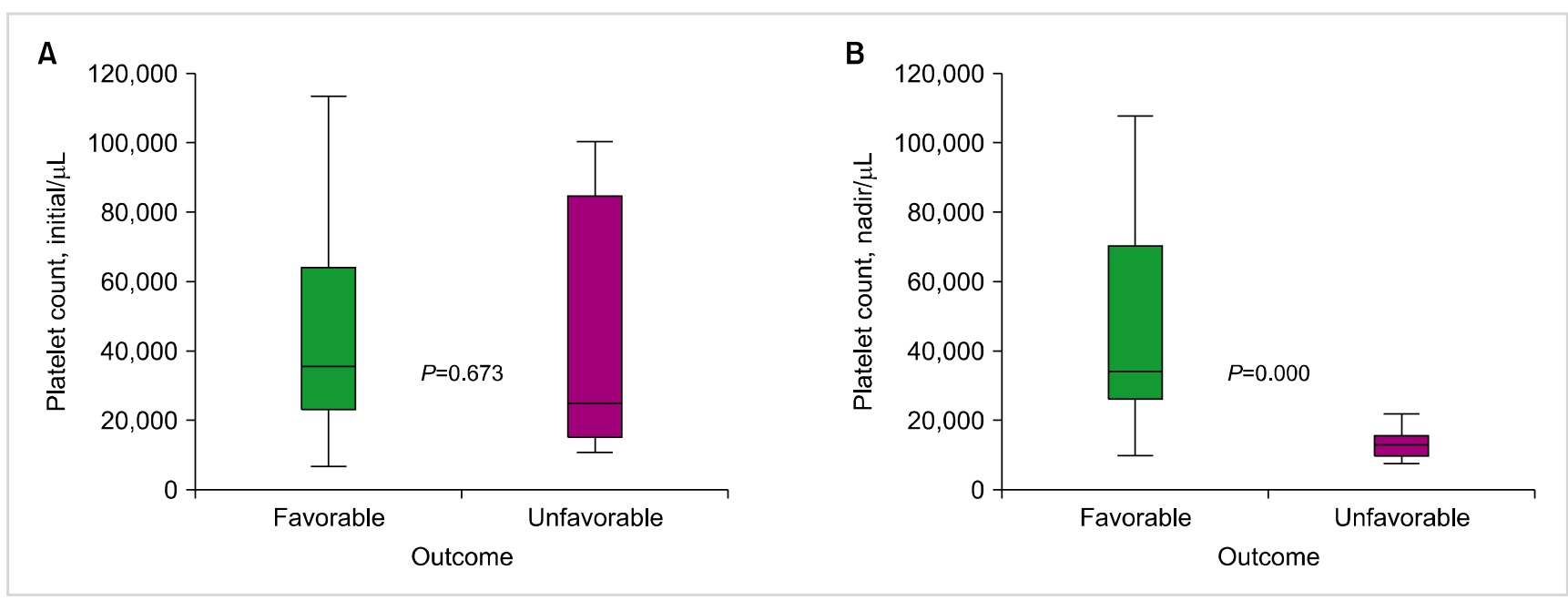

Fig. 1. Comparison of the distribution of the initial (A) and nadir (B) platelet counts between patients with favorable outcomes and those with unfavorable outcomes.

the quantitative value did not correlate with the clinical outcome. Data on peak prothrombin time, activated partial thromboplastin time, and fibrinogen level at nadir were available in our report and two other reports $[1,4]$, revealing disseminated intravascular coagulation features in some patients. However, this finding was not associated with mortality. 


\section{Platelet counts according to clinical outcomes in VITT}

When comparing patients with favorable outcomes (recovery or full recovery) and those with unfavorable outcomes (fatal/died), the initial platelet counts between the groups were not significantly different in 26 patients, as determined using the nonparametric test (median, 35,500 and $23,000 / \mu \mathrm{L}$ ). In contrast, the nadir platelet counts during the clinical course in 10 patients with favorable outcomes were significantly higher than those of 9 patients with

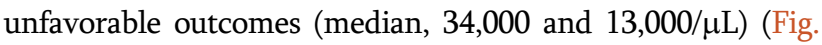
1 ). The cutoff value for predicting fatality based on the nadir platelet count was $22,000 / \mu \mathrm{L}$, as determined using a receiver operating characteristic curve, with an area under the curve of 0.928 (Fig. 2).

\section{Discussion}

In Korea, adenovirus-based COVID-19 vaccines were banned for those in their 20s and permitted only for those $\geq 30$-years-old since April 25, 2021, after performing the risk-benefit assessment when comparing severe hospitalization and mortality rates after COVID-19 with the predicted mortality of VITT [7]. Moreover, an active surveillance system involving a self-monitoring application that enabled early declaration and inspection was maintained for people receiving AstraZeneca and Janssen vaccines. Tests for the PF4-heparin antibody were performed in a central laboratory. Among the 35 suspected thrombocytopenia and/or thrombosis cases, two were confirmed as VITT [8]. The relatively low incidence of two VITT cases among 12.7 million individuals when compared with that in Western countries (348 cases among 14.3 million individuals receiving AstraZeneca vaccination in the $\mathrm{UK}, 32$ cases among 10.2 million individuals receiving Janssen vaccination in the USA) [9, 10] should be considered when preparing the vaccination

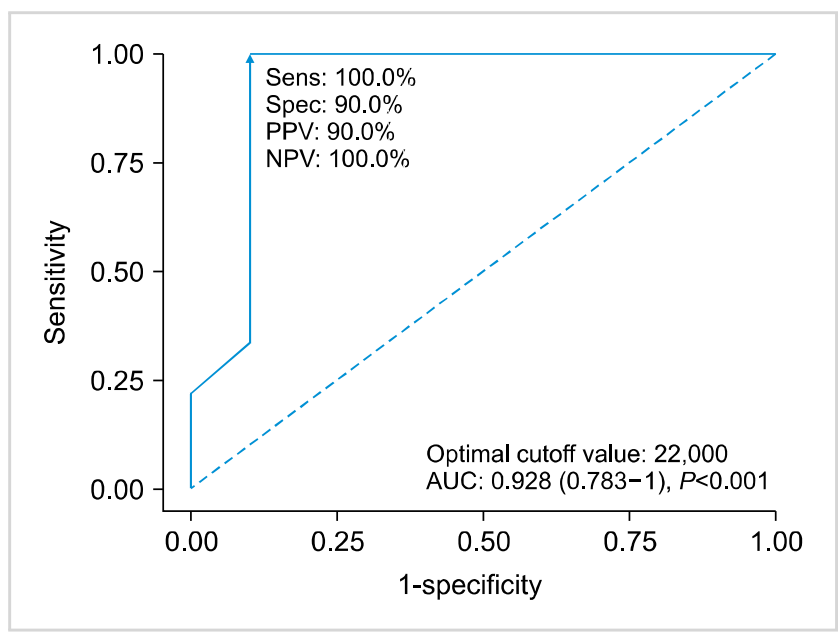

Fig. 2. Receiver operating characteristic curve to predict the prognosis of vaccine-induced immune thrombotic thrombocytopenia (VITT) with the nadir platelet count. guidelines for each country.

Rapid deterioration and fatality due to VITT were the main problems during the early periods, until we elucidated the exact mechanism of the disease. The key pathophysiology causing both thrombosis and bleeding is based on an autoantibody-mediated immune response, similar to heparin-induced thrombocytopenia. Therefore, the induction of immune tolerance through the administration of immunoglobulin and steroids is recommended as an effective treatment. The administration of anticoagulants other than heparin and low-molecular-weight heparin should be considered to improve the clinical course of thrombosis [11]. However, this study revealed that patients with severe thrombocytopenia at presentation or patients who failed to recover their platelet counts during treatment with immunosuppressants and those who bypassed anticoagulants may have a poor prognosis. This is the first important and significant recommendation globally, although only a few VITT cases have been reported, and their numbers are smaller than those reported in each country's media. Our study findings suggest the following recommendations: 1) Individuals who receive AstraZeneca and Janssen vaccines should be educated about visiting the hospital immediately if they experience symptoms possibly related to thrombosis and thrombocytopenia between 4 days and 4 weeks after vaccination (suspected cases). 2) Medical staff in hospitals, including primary clinics, should immediately request PF4/heparin antibody tests to the central laboratory if they screened cases of thrombocytopenia and thrombosis through blood and imaging tests (presumed cases). 3) Patients with thrombocytopenia and thrombosis with positive results for PF4/heparin antibody should be treated with immunoglobulins, steroids, and substitutive anticoagulants (confirmed cases). In conclusion, we cautiously suggest that early diagnosis of VITT after symptom development is the most important aspect in reducing VITT-related fatality. Furthermore, clinicians should consider more active measures, such as plasma exchange, early when they detect patients whose initial or subsequent platelet counts are less than $22,000 / \mu \mathrm{L}$.

\section{Sources of support}

This study was supported by a grant from the Seoul National University Bundang Hospital (Grant No. 16-2017006).

\section{Acknowledgments}

The authors would like to thank the COVID-19 Immunization Safety Management Team of the Korean Disease Control and Prevention Agency and adverse event following immunization response teams of Seoul Metropolitan City and Gyeonggi Province. 


\section{Authors' Disclosures of Potential Conflicts of Interest}

No potential conflicts of interest relevant to this article were reported.

\section{REFERENCES}

1. Greinacher A, Thiele T, Warkentin TE, Weisser K, Kyrle PA, Eichinger S. Thrombotic thrombocytopenia after ChAdOx1 nCov-19 vaccination. N Engl J Med 2021;384:2092-101.

2. Muir KL, Kallam A, Koepsell SA, Gundabolu K. Thrombotic thrombocytopenia after Ad26.COV2.S vaccination. N Engl J Med 2021;384:1964-5.

3. Korea Disease Control and Prevention Agency (KDCA). The current status of Cov-19 vaccination. Cheongju, Korea: KDCA, 2021. (Accessed on June 22, 2021, at http://kdca.go.kr/board/ board.es?mid=a20501010000\&bid=0015\&list_no=713731\&cg code $=\&$ act $=$ view\&nPage $=1$ ).

4. Schultz NH, Sørvoll IH, Michelsen AE, et al. Thrombosis and thrombocytopenia after ChAdOx1 nCoV-19 vaccination. N Engl J Med 2021;384:2124-30.

5. Scully M, Singh D, Lown R, et al. Pathologic antibodies to platelet factor 4 after ChAdOx1 nCoV-19 vaccination. N Engl J Med 2021;384:2202-11.

6. Bourguignon A, Arnold DM, Warkentin TE, et al. Adjunct Immune globulin for vaccine-induced thrombotic thrombo- cytopenia. N Engl J Med 2021. [Epub ahead of print]

7. Korea Disease Control and Prevention Agency (KDCA). Public Health Weekly Report Vol. 14, No. 17, 2021. Cheongju, Korea: KDCA, 2021. (Accessed on June 9, 2021, at https://kdca.go.kr/ board/board.es?mid=a20602010000\&bid=0034\&list_no=713111\& act=view)

8. The diagnosis and treatment guidelines for thrombosis with thrombocytopenia syndrome after Cov-19 vaccination. Cheongju, Korea: Korean Disease Control and Prevention Agency, 2021. (Accessed June 22, 2021, at https://ncv.kdca.go.kr/board.es? mid $=$ a12101000000\&bid $=0031$ \#content .

9. Coronavirus vaccine - weekly summary of Yellow Card reporting. London, UK: Medicines and Healthcare Products Regulatory Agency, 2021. (Accessed June 3, 2021, at https:// www.gov.uk/government/publications/coronavirus-covid-19vaccine-adverse-reactions/coronavirus-vaccine-summary-of-y ellow-card-reporting).

10. Selected adverse events reported after COVID-19 vaccination. Atlanta, GA: Centers for Disease Control and Prevention, 2021. (Accesed May 27, 2021, at https://www.cdc.gov/coronavirus/ 2019-ncov/vaccines/safety/adverse-events.html).

11. Nazy I, Sachs UJ, Arnold DM, et al. Recommendations for the clinical and laboratory diagnosis of VITT against COVID-19: communication from the ISTH SSC Subcommittee on Platelet Immunology. J Thromb Haemost 2021;19:1585-8. 